\title{
Energy Estimates and Decay of Solutions to a Plate Equation with Memory
}

\author{
Yongqin Liu \\ School of Mathematics and Physics, \\ North China Electric Power University, \\ Beijing 102206, China
}

\author{
Jingjing Li \\ School of Mathematics and Physics, \\ North China Electric Power University, \\ Beijing 102206, China
}

\begin{abstract}
This paper focuses on the initial-value problem of a linear plate equation with memory in multi-dimensions $(n \geq 1)$, the decay structure of which is of regularity-loss property. By making use of the point-wise estimate of solutions in the Fourier space, the energy estimates and decay estimates of solutions to the linear problem are obtained.
\end{abstract}

Keywords: plate equation with memory; point-wise estimate; decay estimates; energy estimates

2010 Mathematical Subject Classification Numbers:

35G10;35L30;35B40

\section{INTRODUCTION}

In this paper we consider the initial-value problem of the following linear plate equation with memory term in multidimensional space $\mathbb{R}^{n}$ with $\mathrm{n} \geq 1$ :

$$
u_{t t}+\Delta^{2} u-\Delta u+g * \Delta u=0
$$

with the initial data

$$
u(x, 0)=u_{0}(x), u_{t}(x, 0)=u_{1}(x) .
$$

Here $u=u(x, t)$ is the unknown function of $x=\left(x_{1}, \cdots, x_{n}\right) \in$ $\mathbb{R}^{n}$ and $t>0$, and represents the transversal displacement of the plate at the point $x$ and the time $t$. The term $g * \Delta u=$ $\int_{0}^{t} g(t-\tau) \Delta u(\tau) d \tau$ equivalent to the memory term and $g$ satisfies:

\section{Assumption [1]}

1) $g \in C^{2}\left(\mathbb{R}^{+}\right) \cap W^{2,1}\left(\mathbb{R}^{+}\right)$,

2) $g(s)>0,-C_{0} g(s) \leq g^{\prime}(s) \leq-C_{1} g(s)$,

$\left|g^{\prime \prime}(s)\right| \leq C_{2} g(s), \forall s \in R^{+}$,

3) $1-\int_{0}^{t} g(s) d s \geq C_{3}, \forall t \in \mathbb{R}^{+}$,

where $C_{i}(i=1,2,3)$ are positive constants.

The inertial model of quasilinear dissipative plate equation, whose linear part is given by:

$$
u_{t t}-\Delta u_{t t}+\Delta^{2} u+u_{t}=0
$$

here $-\Delta u_{t t}$ corresponds to the rotational inertia and $u_{t}$ is the linear dissipative term. In [1], Da-Luz and Charão studied a semi-linear dissipative plate equation (1.3). They proved the global existence of solutions and a polynomial decay of the energy by exploiting an energy method. However their result was restricted to the lower dimensional case $1 \leq n \leq 5$. This restriction on the space dimension was removed by Sugitani and Kawashima by making use of the sharp decay estimates for the equation (1.3) in [2]. In [3], Liu and Kawashima obtained the global existence and asymptotic behavior of solutions by employing the time-weighted energy method combined with a semi-group argument. In this paper the plate equation with memory (1.1) is also of regularity-loss property, just like the inertial model of dissipative plate equation $(1.3)$ in $[2,3]$. The decay structure of the regularity-loss is characterized by the property

$$
\rho(\xi)=\frac{|\xi|^{2}}{\left(1+|\xi|^{2}\right)^{2}}
$$

where $\rho(\xi)$ is introduced in the point-wise estimate in the Fourier space of solutions to the linear problem. It is evident that the decay structure is very weak in the higher-frequency domain since $\rho(\xi) \rightarrow 0$ as $\xi \rightarrow \infty$. The decay structure of the regularity-loss type was also observed in $[4,5,6,7]$. For more studies on various aspects of dissipation of plate equations, we refer to $[8,9,10,11]$. Also, as for the study of decay properties for hyperbolic systems of memory-type dissipation, we refer to $[12,13,14,15,16]$.

The main aim of this paper is to study decay estimates of solutions to the initial value problem (1.1) and (1.2). Firstly, by using Fourier transform and Laplace transform, we obtain the solution $u$ to the linear problem (1.1) and (1.2) given by (2.4) and the solution operators $\mathrm{G}(\mathrm{t}) *$ and $\mathrm{H}(\mathrm{t}) *$. Secondly, by employing the energy method in the Fourier space, we obtain the pointwise estimate in the Fourier space of solutions to the linear problem (1.1) and (1.2), appealing to which we obtain the point-wise of solution operators and their properties. Finally, the decay estimates of solutions to (1.1), (1.2) are achieved.

The contents of the paper are as follows. Solution formula are obtained in section 2. In section 3, we obtain the estimates and properties of solutions operators, which is based on the point-wise estimate in the Fourier space of solutions to the linear problem. In the last section, we prove the decay estimates of solutions to the linear problem by virtue of the properties of solution operator.

Before closing this section, we give some notations to be used below.

Let $\mathcal{F}[f]$ denote the Fourier transform of $f$ defined by

$$
\mathcal{F}[f]=\hat{f}(\xi):=\frac{1}{(2 \pi)^{\frac{n}{2}}} \int_{\mathbb{R}^{n}} e^{-i x \cdot \xi} f(x) d x,
$$


and we denote its inverse transform as $\mathcal{F}^{-1}$.

$L^{p}=L^{p}\left(\mathbb{R}^{n}\right)(1 \leq p \leq \infty)$ is the usual Lebesgue space with the norm $\|\cdot\|_{L^{p}} . W^{m, p}\left(\mathbb{R}^{n}\right), m \in Z_{+}, p \in[1, \infty)$ denote the usual Sobolev space with its norm

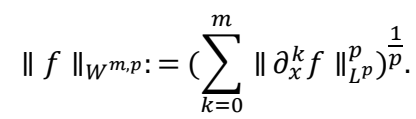

In particular, we use $W^{m, 2}=H^{m}$. Here, for a nonnegative interger $k, \partial_{x}^{k}$ denotes the totality or each of all the $k$-th order derivatives with respect to $x \in \mathbb{R}^{n}$. Also, $C^{k}\left(I ; H^{m}\left(\mathbb{R}^{n}\right)\right)$ denotes the space of $k$-times continuously differentiable functions on the interval $I$ with values in the Sobolev space $H^{m}=H^{m}\left(\mathbb{R}^{n}\right)$.

Finally, in this paper, we denote every positive constant by the same symbol $C$ or $c$ without confusion. [·] is Gauss symbol.

\section{Solution Formula}

In this section, we try to obtain the solution formula for the problem (1.1) and (1.2). Assume that $G(x, t)$ and $H(x, t)$ are the solutions to the following problem:

$$
\begin{aligned}
& \left\{\begin{array}{l}
G_{t t}+\Delta^{2} G-\Delta G+g * \Delta G=0, \\
G(x, 0)=\delta(x), \\
G_{t}(x, 0)=0 .
\end{array}\right. \\
& \left\{\begin{array}{l}
H_{t t}+\Delta^{2} H-\Delta H+g * \Delta H=0, \\
H(x, 0)=0, \\
H_{t}(x, 0)=\delta(x) .
\end{array}\right.
\end{aligned}
$$

Apply Fourier transform and Laplace transform to (2.1) and (2.2), then we can obtain $\widehat{G}(\xi, t)$ and $\widehat{H}(\xi, t)$.

Now we compute $\widehat{G}(\xi, t)$, First, apply Fourier transform to (2.1), we can obtain the following equation:

$$
\left\{\begin{array}{l}
\widehat{G}_{t t}+|\xi|^{4} \hat{G}+|\xi|^{2} \hat{G}-|\xi|^{2} g * \widehat{G}=0, \\
\widehat{G}(\xi, 0)=\hat{\delta}(\xi)=C, \\
\widehat{G}_{t}(\xi, 0)=0
\end{array}\right.
$$

then apply Laplace transform to above equation, we can get

$$
\begin{aligned}
\int_{0}^{\infty} \widehat{G}_{t t} e^{-\lambda t} d t+ & \left(|\xi|^{2}+|\xi|^{4}\right) \int_{0}^{\infty} \widehat{G} e^{-\lambda t} d t-|\xi|^{2} \int_{0}^{\infty}(g \\
& * \widehat{G}) e^{-\lambda t} d t=0
\end{aligned}
$$

by computing, we have that

$$
-C \lambda+\left(\lambda^{2}+|\xi|^{2}+|\xi|^{4}\right) \mathcal{L}[\hat{G}](\lambda)-|\xi|^{2} \mathcal{L}[g](\lambda) \cdot \mathcal{L}[\hat{G}](\lambda)=0,
$$

So

$$
\mathcal{L}[\widehat{G}](\lambda)=\frac{C \lambda}{\lambda^{2}+|\xi|^{2}+|\xi|^{4}-|\xi|^{2} \mathcal{L}[g](\lambda)}
$$

Similarly,

$$
\mathcal{L}[\widehat{H}](\lambda)=\frac{C}{\lambda^{2}+|\xi|^{2}+|\xi|^{4}-|\xi|^{2} \mathcal{L}[g](\lambda)} .
$$

finally, we have formally that

$$
\begin{aligned}
& \widehat{G}(\xi, t)=C \mathcal{L}^{-1}\left[\frac{\lambda}{\lambda^{2}+|\xi|^{2}+|\xi|^{4}-|\xi|^{2} \mathcal{L}[g](\lambda)}\right](\xi, t), \\
& \widehat{H}(\xi, t)=C \mathcal{L}^{-1}\left[\frac{1}{\lambda^{2}+|\xi|^{2}+|\xi|^{4}-|\xi|^{2} \mathcal{L}[g](\lambda)}\right](\xi, t) .
\end{aligned}
$$

Here $C$ is a constant determined by the initial data in (2.1) and (2.2)

Lemma 2.1. $\widehat{G}(\xi, t)$ and $\widehat{H}(\xi, t)$ exist.

Proof. We only prove $\hat{G}(\xi, t)$ exists; similarly we could prove $\widehat{H}(\xi, t) \quad$ exists. Denote $\quad F(\lambda):=\lambda^{2}+|\xi|^{2}+|\xi|^{4}-$ $|\xi|^{2} \mathcal{L}[g](\lambda)$. To prove $\mathcal{L}^{-1}\left[\frac{\lambda}{F(\lambda)}\right]$ exists, we need to consider the zero points of $F(\lambda)$. Denote $\lambda=\sigma+i v, \sigma>-C_{1}, C_{1}$ is same as that in Assumption [1] 2), then $\mathcal{L}[g](\lambda)$ exists. Assume that $\lambda_{1}=\sigma_{1}+i v_{1}$ is a zero point of $F(\lambda)$ and $\sigma_{1}>-C_{1}$, then $\sigma_{1}$ and $v_{1}$ satisfy

$\left\{\begin{array}{l}\operatorname{Re} F\left(\lambda_{1}\right)=\sigma_{1}^{2}-v_{1}^{2}+|\xi|^{2}+|\xi|^{4}-|\xi|^{2} \int_{0}^{\infty} \cos \left(v_{1} t\right) e^{-\sigma_{1} t} g(t) d t=0, \\ \operatorname{Im} F\left(\lambda_{1}\right)=2 \sigma_{1} v_{1}+|\xi|^{2} \int_{0}^{\infty} \sin \left(v_{1} t\right) e^{-\sigma_{1} t} g(t) d t=0 .\end{array}\right.$

If $\xi=0$, from(2.3), we know that $\sigma_{1}=0, v_{1}=0$.

If $\xi \neq 0$, we claim that $\sigma_{1}<0$. Now we prove the claim by contradiction.

Assume that $\sigma_{1} \geq 0$. If $v_{1}=0$, then in view of $\int_{0}^{\infty} g(t) d t<$ 1 , we obtain that

$$
\operatorname{Re} F\left(\lambda_{1}\right)=\sigma_{1}^{2}+|\xi|^{2}+|\xi|^{4}-|\xi|^{2} \int_{0}^{\infty} e^{-\sigma_{1} t} g(t) d t>0
$$

it yields contradiction with $(2.3)_{1}$.

If $v_{1} \neq 0$, then we have that

$$
\operatorname{Im} F\left(\lambda_{1}\right)=v_{1}\left(2 \sigma_{1}+|\xi|^{2} \int_{0}^{\infty} \frac{\sin \left(v_{1} t\right)}{v_{1}} e^{-\sigma_{1} t} g(t) d t\right) .
$$

Next we prove that $\int_{0}^{\infty} \frac{\sin \left(v_{1} t\right)}{v_{1}} e^{-\sigma_{1} t} g(t) d t>0$. Denote $a_{m}=$ $\int_{0}^{\frac{2 m \pi}{\left|v_{1}\right|}} \frac{\sin \left|v_{1} t\right|}{\left|v_{1}\right|} e^{-\sigma_{1} t} g(t) d t$, and we will prove $\left\{a_{m}\right\}_{m=1}^{\infty}$ is a convergent sequence. By direct computation, we have that

$$
a_{1}=\int_{0}^{\frac{\pi}{\left|v_{1}\right|}} \frac{\sin \left|v_{1} t\right|}{\left|v_{1}\right|}\left(e^{-\sigma_{1} t} g(t)-e^{-\sigma_{1}\left(t+\frac{\pi}{\left|v_{1}\right|}\right)} g\left(t+\frac{\pi}{\left|v_{1}\right|}\right)\right) d t \text {. }
$$

Since $\partial_{t}\left(e^{-\sigma_{1} t} g(t)\right)<0$, we have that $0<a_{1}<$ $\int_{0}^{\frac{\pi}{\left|\nu_{1}\right|}} t e^{-\sigma_{1} t} g(t) d t$. Similarly,

$$
a_{m+1}-a_{m}=\int_{\frac{2 m \pi}{\left|v_{1}\right|}}^{\frac{2 m \pi+\pi}{\left|v_{1}\right|}} \frac{\sin \left|v_{1} t\right|}{\left|v_{1}\right|}\left(e^{-\sigma_{1} t} g(t)-e^{-\sigma_{1}\left(t+\frac{\pi}{\left|v_{1}\right|}\right)} g\left(t+\frac{\pi}{\left|v_{1}\right|}\right)\right) d t,
$$

so we have that $0<a_{m+1}-a_{m}<\int_{\frac{2 m \pi}{\left|v_{1}\right|}}^{\frac{2 m \pi+\pi}{\left|v_{1}\right|}} t e^{-\sigma_{1} t} g(t) d t$.

It yields that

$$
0<a_{m}<\int_{0}^{\frac{2 m \pi}{\left|v_{1}\right|}} t e^{-\sigma_{1} t} g(t) d t \leq \frac{g(0)}{\left(\sigma_{1}+C_{1}\right)^{2}}
$$

so $\left\{a_{m}\right\}_{m=1}^{\infty}$ is a bounded and monotonic increasing sequence. Since $a_{1}>0, a\left(\lambda_{1}\right):=\lim _{m \rightarrow \infty} a_{m}>0$. Thus we proved that 
$\int_{0}^{\infty} \frac{\sin \left|v_{1} t\right|}{\left|v_{1}\right|} e^{-\sigma_{1} t} g(t) d t>0$. Also, because $\sigma_{1} \geq 0, \xi \neq 0$ and $v_{1} \neq 0$, it results that $\operatorname{Im} F\left(\lambda_{1}\right) \neq 0$. This contradicts with $(2.3)_{2}$. Thus by contradiction we proved the claim $\sigma_{1}<0$.

Combining the two cases, we know that $\frac{\lambda}{F(\lambda)}$ is analytic in $\{\lambda \in \mathbb{C} ; \operatorname{Re}(\lambda)>0\}$ if $\xi=0$ and in $\{\lambda \in \mathbb{C} ; \operatorname{Re}(\lambda) \geq 0\}$ if $\xi \neq$ 0 . Take $\lambda=\sigma+i v, \sigma>\max \left\{\operatorname{Re} \lambda_{s}\right\}$, here $\left\{\lambda_{s}\right\}$ is the set of all the singular points of $F(\lambda)$, then we have that

$$
\begin{aligned}
\mathcal{L}^{-1}\left[\frac{\lambda}{F(\lambda)}\right](t) & =\int_{\sigma-i \infty}^{\sigma+i \infty} \frac{\lambda e^{\lambda t}}{F(\lambda)}=\int_{-\infty}^{+\infty} \frac{i(\sigma+i v) e^{(\sigma+i v) t}}{F(\sigma+i v)} d v \\
= & \int_{\{v ;|v| \leq R\}}+\int_{\{v ;|v|>R\}}=: J_{1}+J_{2}
\end{aligned}
$$

$J_{1}$ converges, so we only need to consider $J_{2}$. Notice that $\frac{\lambda}{F(\lambda)}=$ $\frac{1}{\lambda}-\frac{|\xi|^{2}+|\xi|^{4}-|\xi|^{2} \mathcal{L}[g](\lambda)}{\lambda F(\lambda)}$ and $|\mathcal{L}[g](\lambda)| \leq C$, then it is not difficult to prove that $J_{2}$ converges. The constant $C$ in the expression of $\hat{G}(\xi, t)$ is determined by the initial data of $G(x, t)$. So far we complete the proof.

In view of Lemma 2.1 and Duhamel principle, the solution to the problem (1.1) and (1.2) could be expressed as following:

$$
u(t)=G(t) * u_{0}+H(t) * u_{1}
$$

\section{Decay Properties of Solution Operators}

In this section, our goal is to get the decay estimates of the solution operators $G(t) *$ and $H(t) *$ in the solution formula (2.4).

Proposition 3.1. Let $k$ and $l$ be integers, $\varphi \in$ $H^{s+1}\left(\mathbb{R}^{n}\right) \cap L^{p}\left(\mathbb{R}^{n}\right), \psi \in H^{s-1}\left(\mathbb{R}^{n}\right) \cap L^{p}\left(\mathbb{R}^{n}\right), 1 \leq p \leq 2$, then the following estimates hold:

$$
\text { 1) } \begin{aligned}
\left\|\partial_{x}^{k} G(t) * \varphi\right\|_{L^{2}} \leq & C(1+t)^{-\frac{k}{2}-\frac{n}{2}\left(\frac{1}{p}-\frac{1}{2}\right)}\|\varphi\|_{L^{p}} \\
& +C(1+t)^{-\frac{l}{2}}\left\|\partial_{x}^{k+l} \varphi\right\|_{L^{2}},
\end{aligned}
$$

for $k \geq 0, l \geq 0, l+k \leq s+1$.

$$
\begin{aligned}
& \text { 2) }\left\|\partial_{x}^{k} G_{t}(t) * \varphi\right\|_{L^{2}} \leq C(1+t)^{-\frac{k+1}{2}-\frac{n}{2}\left(\frac{1}{p}-\frac{1}{2}\right)}\|\varphi\|_{L^{p}} \\
& +C(1+t)^{-\frac{l}{2}}\left\|\partial_{x}^{k+l+2} \varphi\right\|_{L^{2}} \text {, for } k \geq 0,
\end{aligned}
$$

$l \geq 0, l+k \leq s-1$.

$$
\begin{aligned}
& \text { 3) }\left\|\partial_{x}^{k} H(t) * \psi\right\|_{L^{2}} \leq C(1+t)^{-\frac{k-1}{2}-\frac{n}{2}\left(\frac{1}{p}-\frac{1}{2}\right)}\|\psi\|_{L^{p}} \\
& +C(1+t)^{-\frac{l+2}{2}}\left\|\partial_{x}^{k+l} \psi\right\|_{L^{2}},
\end{aligned}
$$

for $k \geq 1, l+2 \geq 0,0 \leq l+k \leq s-1$.

$$
\text { 4) } \begin{aligned}
\left\|\partial_{x}^{k} H_{t}(t) * \psi\right\|_{L^{2}} \leq & C(1+t)^{-\frac{k}{2}-\frac{n}{2}\left(\frac{1}{p}-\frac{1}{2}\right)}\|\psi\|_{L^{p}} \\
& +C(1+t)^{-\frac{l}{2}}\left\|\partial_{x}^{k+l} \psi\right\|_{L^{2}},
\end{aligned}
$$

for $k \geq 0, l \geq 0, l+k \leq s-1$.

To prove proposition 3.1, the key point is to obtain the point-wise estimates of the fundamental solutions in the Fourier space. In fact this could be achieved by using the following point-wise estimate of solutions to the linear problem (1.1) and (1.2).
Lemma 3.1. Assume $u$ is the solution of (1.1) and (1.2), then it satisfies the following point-wise estimate in the Fourier space:

$$
\begin{aligned}
& \left|\hat{u}_{t}(\xi, t)\right|^{2}+\left(|\xi|^{2}+|\xi|^{4}\right)|\hat{u}(\xi, t)|^{2}+|\xi|^{2}(g \square \hat{u})(\xi, t) \\
& \leq C e^{-C \rho(\xi) t}\left(\left|\hat{u}_{1}(\xi)\right|^{2}+\left(|\xi|^{2}+|\xi|^{4}\right)\left|\hat{u}_{0}(\xi)\right|^{2}\right),
\end{aligned}
$$

here $\rho(\xi)=\frac{|\xi|^{2}}{\left(1+|\xi|^{2}\right)^{2}}$.

To prove Lemma 3.1, we denote some notations. For any real or complexvalued function $f(t)$, we define

$$
\begin{gathered}
(g * f)(t):=\int_{0}^{t} g(t-\tau) f(\tau) d \tau, \\
(g \circ f)(t):=\int_{0}^{t} g(t-\tau)(f(\tau)-f(t)) d \tau, \\
(g \square f)(t):=\int_{0}^{t} g(t-\tau)|f(t)-f(\tau)|^{2} d \tau .
\end{gathered}
$$

We have the following lemma rely on direct calculation, which is useful in obtaining our point-wise estimate of solution in the Fourier space.

Lemma 3.2. For any function $\mathrm{k} \in \mathrm{C}(\mathbb{R})$, and any $\phi \in$ $\mathrm{W}^{1,2}(0, \mathrm{~T})$, it holds that

$$
\begin{array}{r}
\text { 1) }(k * \phi)(t)=(k \diamond \phi)(t)+\left(\int_{0}^{t} k(\tau) d \tau\right) \phi(t), \\
\text { 2) } 2 \operatorname{Re}\left\{(k * \phi)(t) \bar{\phi}_{t}(t)\right\}=-k(t)|\phi(t)|^{2}+\left(k^{\prime} \square \phi\right)(t)- \\
\frac{d}{d t}\left\{(k \square \phi)(t)-\left(\int_{0}^{t} k(\tau) d \tau\right)|\phi(t)|^{2}\right\},
\end{array}
$$

3) $|(k \diamond \phi)(t)|^{2} \leq\left(\int_{0}^{t}|k(\tau)| d \tau\right)(|k| \square \phi)(t)$.

Next we will come to get the point-wise estimates of solutions to the problem (1.1), (1.2) in the Fourier space.

Proof of Lemma 3.1

Step 1: Apply Fourier transform to (1.1), we have that

$$
\hat{u}_{t t}+\left(|\xi|^{2}+|\xi|^{4}\right) \hat{u}-|\xi|^{2} g * \hat{u}=0
$$

By multiplying (3.2) by $\overline{\hat{u}}_{t}$ and taking the real part, we have that

$$
\left\{\frac{1}{2}\left|\hat{u}_{t}\right|^{2}+\frac{1}{2}\left(|\xi|^{2}+|\xi|^{4}\right)|\hat{u}|^{2}\right\}_{t}-|\xi|^{2} \operatorname{Re}\left\{g * \hat{u} \overline{\hat{u}}_{t}\right\}=0 .
$$

Use Lemma 3.2. 2) To the term $\operatorname{Re}\left\{g * \hat{u} \overline{\hat{u}}_{t}\right\}$ in (3.3), we have that

$$
\begin{array}{r}
\operatorname{Re}\left\{g * \hat{u} \overline{\hat{u}}_{t}\right\}=-\frac{1}{2} g(t)|\hat{u}|^{2}+\frac{1}{2}\left(g^{\prime} \square \hat{u}\right)(t) \\
-\frac{1}{2} \frac{d}{d t}\left\{(g \square \hat{u})(t)-\int_{0}^{t} g(\tau) d \tau|\hat{u}|^{2}\right\} .
\end{array}
$$

We denote

$$
\begin{gathered}
E_{1}(\xi, t)=\left|\hat{u}_{t}\right|^{2}+\left(|\xi|^{2}+|\xi|^{4}\right)|\hat{u}|^{2}+|\xi|^{2} g \square \hat{u} \\
-|\xi|^{2}\left(\int_{0}^{t} g(s) d s\right)|\hat{u}|^{2}, \\
F_{1}(\xi, t)=|\xi|^{2}\left(g|\hat{u}|^{2}-g^{\prime} \square \hat{u}\right),
\end{gathered}
$$

then we have that

$$
\frac{\partial}{\partial t} E_{1}(\xi, t)+F_{1}(\xi, t)=0 .
$$

Step 2: By multiplying (3.2) by $\left\{-(g * \overline{\hat{u}})_{t}\right\}$ and taking the real part, we have that

$\left\{\frac{1}{2}|\xi|^{2}|g * \hat{u}|^{2}\right\}_{t}-\operatorname{Re}\left\{\hat{u}_{t t}(g * \overline{\hat{u}})_{t}\right\}-$ 


$$
\operatorname{Re}\left\{\left(|\xi|^{2}+|\xi|^{4}\right) \hat{u}(g * \overline{\hat{u}})_{t}\right\}=0
$$

Since $(g * \overline{\hat{u}})_{t}=g(0) \overline{\hat{u}}+g^{\prime} * \overline{\hat{u}}$, the second term in (3.5) yields that

$$
\begin{aligned}
-\operatorname{Re}\left\{\hat{u}_{t t}(g * \overline{\hat{u}})_{t}\right\} & =-\operatorname{Re}\left\{\hat{u}_{t}(g * \overline{\hat{u}})_{t}\right\}_{t}+\operatorname{Re}\left\{\hat{u}_{t}(g * \overline{\hat{u}})_{t t}\right\} \\
& =-\operatorname{Re}\left\{\hat{u}_{t}(g * \hat{\hat{u}})_{t}\right\}_{t}+\operatorname{Re}\left\{\hat{u}_{t}\left(g(0) \hat{\hat{u}}_{t}+\left(g^{\prime} * \overline{\hat{u}}\right)_{t}\right)\right\} \\
& =-\operatorname{Re}\left\{\hat{u}_{t}(g * \overline{\hat{u}})_{t}\right\}_{t}+\operatorname{Re}\left\{g(0)\left|\hat{u}_{t}\right|^{2}+\hat{u}_{t}\left(g^{\prime} * \hat{\hat{u}}\right)_{t}\right\} .
\end{aligned}
$$

We denote

$$
\begin{gathered}
E_{2}(\xi, t)=\frac{1}{2}|\xi|^{2}|g * \hat{u}|^{2}-\operatorname{Re}\left\{\hat{u}_{t}(g * \overline{\hat{u}})_{t}\right\}, \\
F_{2}(\xi, t)=g(0)\left|\hat{u}_{t}\right|^{2}, \\
R_{2}(\xi, t)=\operatorname{Re}\left\{-\hat{u}_{t}\left(g^{\prime} * \overline{\hat{u}}\right)_{t}+\left(|\xi|^{2}+|\xi|^{4}\right) \hat{u}(g * \overline{\hat{u}})_{t}\right\},
\end{gathered}
$$

then obtain that

$$
\frac{\partial}{\partial t} E_{2}(\xi, t)+F_{2}(\xi, t)=R_{2}(\xi, t) .
$$

Step 3: By multiplying (3.2) by $\bar{u}$ and taking the real part, we have that

$$
\operatorname{Re}\left\{\hat{u}_{t} \overline{\hat{u}}_{t}-\left|\hat{u}_{t}\right|^{2}+\left(|\xi|^{2}+|\xi|^{4}\right)|\hat{u}|^{2}-|\xi|^{2} \operatorname{Re}\{g * \hat{u} \overline{\hat{u}}\}=0 .\right.
$$

Due to Lemma 3.21 ), we obtain that

$$
\operatorname{Re}\{g * \hat{u} \bar{u}\}=\left(\int_{0}^{t} g(s) d s\right)|\hat{u}|^{2}+\operatorname{Re}\{g \diamond \hat{u} \overline{\hat{u}}\} .
$$

We denote

$$
\begin{gathered}
E_{3}(\xi, t)=\operatorname{Re}\left\{\hat{u}_{t} \overline{\hat{u}}\right\}, \\
F_{3}(\xi, t)=\left(|\xi|^{2}+|\xi|^{4}\right)|\hat{u}|^{2}-|\xi|^{2}\left(\int_{0}^{t} g(s) d s\right)|\hat{u}|^{2}, \\
R_{3}(\xi, t)=\left|\hat{u}_{t}\right|^{2}+|\xi|^{2} \operatorname{Re}\{g \diamond \hat{u} \overline{\hat{u}}\},
\end{gathered}
$$

Then (3.7) yields that

$$
\frac{\partial}{\partial t} E_{3}(\xi, t)+F_{3}(\xi, t)=R_{3}(\xi, t)
$$

Define $\rho(\xi)=\frac{|\xi|^{2}}{\left(1+|\xi|^{2}\right)^{2}}$, and denote

$$
\begin{gathered}
E(\xi, t)=E_{1}(\xi, t)+\rho(\xi)\left(\alpha E_{2}(\xi, t)+\beta E_{3}(\xi, t)\right), \\
F(\xi, t)=F_{1}(\xi, t)+\rho(\xi)\left(\alpha F_{2}(\xi, t)+\beta F_{3}(\xi, t)\right), \\
R(\xi, t)=\rho(\xi)\left(\alpha R_{2}(\xi, t)+\beta R_{3}(\xi, t)\right),
\end{gathered}
$$

where $\alpha, \beta$ are positive constants, then (3.4), (3.6) and (3.8) yield that

$$
\frac{\partial}{\partial t} E(\xi, t)+F(\xi, t)=R(\xi, t) .
$$

We introduce Lyapunov functions:

$$
\begin{aligned}
E_{0}(\xi, t)= & \left|\hat{u}_{t}\right|^{2}+\left(|\xi|^{2}+|\xi|^{4}\right)|\hat{u}|^{2}+|\xi|^{2} g \square \hat{u} . \\
& F_{0}(\xi, t)=g \square \hat{u}+g|\hat{u}|^{2} .
\end{aligned}
$$

By the definitions of $E_{1}(\xi, t)$ and $F_{1}(\xi, t)$, we know that there exist some positive constants $c_{i}(\mathrm{i}=1,2,3)$ such that the following inequalities hold:

$c_{1} E_{0}(\xi, t) \leq E_{1}(\xi, t) \leq c_{2} E_{0}(\xi, t), F_{1}(\xi, t) \geq c_{3}|\xi|^{2} F_{0}(\xi, t)$.

On the other hand,

$$
\begin{aligned}
\left|E_{2}(\xi, t)\right| \leq C\left|\hat{u}_{t}\right|^{2}+C\left(1+|\xi|^{2}\right)\left(|\hat{u}|^{2}+g \square \hat{u}\right), \\
\left|E_{3}(\xi, t)\right| \leq C\left(\left|\hat{u}_{t}\right|^{2}+|\hat{u}|^{2}\right) \\
\left|\rho(\xi)\left(\alpha E_{2}(\xi, t)+\beta E_{3}(\xi, t)\right)\right| \\
\leq C(\alpha+\beta)\left\{\left|\hat{u}_{t}\right|^{2}+|\xi|^{2}\left(|\hat{u}|^{2}+g \square \hat{u}\right)\right\} \\
\leq c_{4}(\alpha+\beta) E_{0}(\xi, t) .
\end{aligned}
$$

Choose $\alpha, \beta$ appropriately small such that $c_{4}(\alpha+\beta) \leq$ $\min \left(\frac{c_{1}}{2}, \frac{c_{2}}{2}\right)$, from $(3.10)$ we have that

$$
\frac{c_{1}}{2} E_{0}(\xi, t) \leq E(\xi, t) \leq \frac{3 c_{2}}{2} E_{0}(\xi, t) .
$$

In consideration of (3.10) and the fact that $0 \leq \int_{0}^{t} g(s) d s \leq$ 1 , it is not difficult to verify that

$$
F(\xi, t) \geq c_{3}|\xi|^{2} F_{0}(\xi, t)+\rho(\xi)\left\{\alpha g(0)\left|\hat{u}_{t}\right|^{2}+\beta|\xi|^{4}|\hat{u}|^{2}\right\} .
$$

By virtue of Lemma 3.2 , we have that

$$
\begin{aligned}
\left|R_{2}(\xi, t)\right| \leq & \varepsilon\left|\hat{u}_{t}\right|^{2}+\delta\left(|\xi|^{2}+|\xi|^{4}\right)|\hat{u}|^{2} \\
& +C_{\varepsilon, \delta}\left(|\xi|^{2}+|\xi|^{4}\right) F_{0}(\xi, t)
\end{aligned}
$$

and

$$
\left|R_{3}(\xi, t)\right| \leq\left|\hat{u}_{t}\right|^{2}+\gamma|\xi|^{2}|\hat{u}|^{2}+C_{\gamma}|\xi|^{2} g \square \hat{u},
$$

where $\varepsilon, \delta, \gamma$ are positive constants, we have

$$
\begin{aligned}
|R(\xi, t)| & \leq \rho(\xi)\left\{(\alpha \varepsilon+\beta)\left|\hat{u}_{t}\right|^{2}+(\alpha \delta+\beta \gamma)\left(|\xi|^{2}+|\xi|^{4}\right)|\hat{u}|^{2}\right. \\
& \left.+\alpha C_{\varepsilon, \delta}\left(|\xi|^{2}+|\xi|^{4}\right) F_{0}(\xi, t)+\beta C_{\gamma}|\xi|^{2} g \square \hat{u}\right\} \\
& \leq(\alpha \varepsilon+\beta) \rho(\xi)\left|\hat{u}_{t}\right|^{2}+(\alpha \delta+\beta \gamma)|\xi|^{2}|\hat{u}|^{2}+(\alpha+\beta) C_{\varepsilon, \delta, \gamma}|\xi|^{2} F_{0}(\xi, t) .
\end{aligned}
$$

We claim that there exist $\gamma, \varepsilon, \delta, \alpha, \beta$ such that

$$
|R(\xi, t)| \leq \frac{1}{2} F(\xi, t) .
$$

First choose $\gamma=\frac{1}{4}, \varepsilon=\frac{1}{4} g(0), \delta=\frac{1}{16} g(0), \beta=\frac{1}{4} \alpha g(0)$, then the next three inequalities hold:

$$
\begin{gathered}
(\alpha+\beta) C_{\varepsilon, \delta, \gamma} \leq \frac{1}{2} c_{3}, \\
\alpha \varepsilon+\beta \leq \frac{1}{2} \alpha g(0), \\
\alpha \delta+\beta \gamma \leq \frac{1}{2} \beta,
\end{gathered}
$$

In order to certify (3.13) (here (3.11) is also considered), it suffices to choose $\alpha$ suitably small such that

$$
\alpha+\beta=\left(1+\frac{1}{4} g(0)\right) \alpha \leq \min \left\{\frac{c_{3}}{2 C_{\varepsilon, \delta, \gamma}}, \frac{c_{1}}{2 c_{4}}, \frac{c_{2}}{2 c_{4}}\right\}
$$

Due to (3.9) and (3.13), we get that

$$
\frac{\partial}{\partial t} E(\xi, t)+\frac{1}{2} F(\xi, t) \leq 0 .
$$

On the other hand, due to (3.11) and (3.12) we obtain that

$$
F(\xi, t)>c \rho(\xi) E(\xi, t) \text {. }
$$

From (3.14) and (3.15), we have that

$$
E(\xi, t) \leq e^{-C \rho(\xi) t} E(\xi, 0) .
$$

By virtue of (3.11) and (3.16), we have that

$$
\left|\hat{u}_{t}\right|^{2}+\left(|\xi|^{2}+|\xi|^{4}\right)|\hat{u}|^{2}+|\xi|^{2} g \square \hat{u} \leq
$$




$$
C e^{-C \rho(\xi) t}\left(\left|\hat{u}_{1}(\xi)\right|^{2}+\left(|\xi|^{2}+|\xi|^{4}\right)\left|\hat{u}_{0}(\xi)\right|^{2}\right),
$$

so, we obtain the point-wise estimates of solutions to (1.1), (1.2) in the Fourier space.

As a simple corollary of Lemma 3.1, we have the following point-wise estimates of the fundamental solutions $G(x, t)$ and $H(x, t)$ in the Fourier space.

Lemma 3.3. $G(x, t)$ and $H(x, t)$ satisfy

1) $|\widehat{G}(\xi, t)| \leq C e^{-C \rho(\xi) t}$,

2) $\left|\widehat{G}_{t}(\xi, t)\right| \leq C e^{-C \rho(\xi) t}\left(|\xi|^{2}+|\xi|^{4}\right)^{\frac{1}{2}}$,

3) $|\widehat{H}(\xi, t)| \leq C e^{-C \rho(\xi) t}\left(|\xi|^{2}+|\xi|^{4}\right)^{-\frac{1}{2}}$,

4) $\left|\widehat{H}_{t}(\xi, t)\right| \leq C e^{-C \rho(\xi) t}$,

where $\rho(\xi)=\frac{|\xi|^{2}}{\left(1+|\xi|^{2}\right)^{2}}$.

Proof. Putting (2.4) with $u_{1}=0$ in (3.1), it results that

$\left|\widehat{G}_{t}(\xi, t)\right|^{2}+\left(|\xi|^{2}+|\xi|^{4}\right)|\widehat{G}(\xi, t)|^{2} \leq C e^{-C \rho(\xi) t}\left(|\xi|^{2}+|\xi|^{4}\right)$.

It yields 1) and 2) of Lemma 3.3.

Putting (2.4) with $u_{0}=0$ in (3.1), it results that

$$
\left|\widehat{H}_{t}(\xi, t)\right|^{2}+\left(|\xi|^{2}+|\xi|^{4}\right)|\widehat{H}(\xi, t)|^{2} \leq C e^{-C \rho(\xi) t} .
$$

It yields 3) and 4) of Lemma 3.3.

Now we use Lemma 3.3 to prove Proposition 3.1.

Proof of Proposition 3.1. In view of Lemma 3.3 1), we have that

$$
\begin{aligned}
& \left\|\partial_{x}^{k} G_{t}(t) * \varphi\right\|_{L^{2}}^{2} \leq C \int_{R^{n}}|\xi|^{2 k} e^{-C \rho(\xi) t}|\hat{\varphi}(\xi)|^{2} d \xi \\
\leq & C \int_{\{\xi:|\xi| \leq 1\}}|\xi|^{2 k} e^{-\frac{C}{4}|\xi|^{2} t}|\hat{\varphi}|^{2} d \xi+C \int_{\{\xi:|\xi| \geq 1\}}|\xi|^{2 k} e^{-\frac{C t}{4|\xi|^{2}}|\hat{\varphi}(\xi)|^{2} d \xi} \\
\leq & C(1+t)^{-n\left(\frac{1}{p}-\frac{1}{2}\right)-k}\|\varphi\|_{L^{p}}^{2}+C(1+t)^{-l}\left\|\partial_{x}^{k+l} \varphi\right\|_{L^{2}}^{2},
\end{aligned}
$$

here $k \geq 0, l \geq 0, l+k \leq s+1$. Thus 1 ) is proved.

Due to Lemma 3.3 2), it results that

$$
\begin{aligned}
& \left\|\partial_{x}^{k} G_{t}(t) * \varphi\right\|_{L^{2}}^{2} \leq C \int_{R^{n}}|\xi|^{2 k}\left(|\xi|^{2}+|\xi|^{4}\right) e^{-C \rho(\xi) t}|\hat{\varphi}(\xi)|^{2} d \xi \\
\leq & C \int_{\{\xi: \xi \mid \leq 1\}}|\xi|^{2 k+2} e^{-\frac{C}{4}|\xi|^{2} t}|\hat{\varphi}(\xi)|^{2} d \xi+C \int_{\{\xi:|\xi| \geq 1\}}|\xi|^{2 k+4} e^{-\frac{C t}{4|\xi|^{2}}}|\hat{\varphi}(\xi)|^{2} d \xi \\
\leq & C(1+t)^{-n\left(\frac{1}{p}-\frac{1}{2}\right)-k-1}\|\varphi\|_{L^{p}}^{2}+C(1+t)^{-l}\left\|\partial_{x}^{k+l+2} \varphi\right\|_{L^{2}}^{2},
\end{aligned}
$$

here $k+1 \geq 0, l \geq 0, k+l+2 \leq s+1$. Thus 2) is proved. that

Next we prove 3 ) and 4 ). It follows from Lemma 3.3 3)

$$
\begin{aligned}
& \left\|\partial_{x}^{k} H(t) * \psi\right\|^{2} \leq C \int_{R^{n}}|\xi|^{2 k}\left(|\xi|^{2}+|\xi|^{4}\right)^{-1} e^{-C \rho(\xi) t}|\hat{\psi}(\xi)|^{2} d \xi \\
\leq & C \int_{\{\xi:|\xi| \leq 1\}}|\xi|^{2 k-2} e^{-\frac{C}{4}|\xi|^{2} t}|\hat{\psi}(\xi)|^{2} d \xi+C \int_{\{\xi: \xi \mid \geq 1\}}|\xi|^{2 k}|\xi|^{-4} e^{-\frac{C t}{4|\xi|}}|\hat{\psi}(\xi)|^{2} d \xi \\
\leq & C(1+t)^{-n\left(\frac{1}{p}-\frac{1}{2}\right)-k+1}\|\psi\|_{L^{p}}^{2}+C(1+t)^{-l-2}\left\|\partial_{x}^{k+l} \psi\right\|_{L^{2}}^{2},
\end{aligned}
$$

here $k \geq 1, l \geq-2,0 \leq k+l \leq s-1$. Thus 3 ) is proved.

Lemma 3.32 ), yields that

$$
\begin{gathered}
\left\|\partial_{x}^{k} H_{t}(t) * \psi\right\|^{2} \leq C \int_{R^{n}}|\xi|^{2 k} e^{-C \rho(\xi) t}|\widehat{\psi}(\xi)|^{2} d \xi \\
\leq C(1+t)^{-n\left(\frac{1}{p}-\frac{1}{2}\right)-k}\|\psi\|_{L^{p}}^{2}+C(1+t)^{-l}\left\|\partial_{x}^{k+l} \psi\right\|_{L^{2}}^{2}
\end{gathered}
$$

here $k \geq 0, l \geq 0, k+l \leq s-1$. thus 4 ) is proved.

\section{Decay Estimates for Linear Problem.}

In this section we study the decay estimates of solutions to the linear problem (1.1) and (1.2).

Theorem 4.1. Let $s \geq 1$ be an integer. Assume that $u_{0} \in$ $H^{s+1}\left(\mathbb{R}^{n}\right)$ and $u_{1} \in H^{s-1}\left(\mathbb{R}^{n}\right)$, and put

$$
I_{0}=\left\|u_{0}\right\|_{H^{s+1}}+\left\|u_{1}\right\|_{H^{s-1}} .
$$

Then the solution $u$ to the problem (1.1) and (1.2) given by (2.4) satisfies

$$
\left.\partial_{x} u \in C^{0}([0, \infty)) ; H^{s}\left(\mathbb{R}^{n}\right)\right) ; u \in C^{1}\left([0, \infty) ; H^{s-1}\left(\mathbb{R}^{n}\right)\right)
$$

and the following energy estimate:

$\left\|u_{t}(t)\right\|_{H^{s-1}}^{2}+\left\|\partial_{x} u\right\|_{H^{s}}^{2}+\int_{0}^{t}\left\|\partial_{x} u_{t}(\tau)\right\|_{H^{s-3}}^{2}+\left\|\partial_{x}^{2} u(\tau)\right\|_{H^{s-2}}^{2} d \tau \leq C I_{0}^{2}$.

Proof. We have obtained the solution $u$ of (1.1) and (1.2) given by (2.4) and proved that it satisfies the point-wise estimate (3.1) in the Fourier space.

From (3.14) and (3.15) we have that

$$
\frac{\partial}{\partial t} E(\xi, t)+C \rho(\xi) E(\xi, t) \leq 0 .
$$

Integrate the inequality with respect to $t$ and appeal to (3.11 ), then we obtain

$$
E_{0}(\xi, t)+\int_{0}^{t} \rho(\xi) E_{0}(\xi, \tau) d \tau \leq C E_{0}(\xi, t) .
$$

Multiply (4.1) by $\left(1+|\xi|^{2}\right)^{s-1}$ and integrate the resulting inequality with respect to $\xi \in \mathbb{R}^{\mathrm{n}}$, then we have that

$\left\|u_{t}(t)\right\|_{H^{s-1}}^{2}+\left\|\partial_{\mathrm{x}} \mathrm{u}\right\|_{\mathrm{H}^{s}}^{2}+\int_{0}^{\mathrm{t}}\left\|\partial_{\mathrm{x}} \mathrm{u}_{\mathrm{t}}(\tau)\right\|_{\mathrm{H}^{s-3}}^{2}+\left\|\partial_{\mathrm{x}}^{2} \mathrm{u}(\tau)\right\|_{\mathrm{H}^{\mathrm{s}-2}}^{2} \mathrm{~d} \tau \leq \mathrm{CI}_{0}^{2}$.

(4.2) guarantees the regularity of the solution (2.4). So far we complete the proof of Theorem 4.1 .

By using Proposition 3.1 with $\mathrm{p}=2$, we obtain the following decay estimate of $u$ given by (2.4), if initial data $u_{0} \in$ $\mathrm{H}^{\mathrm{s}+1}\left(\mathbb{R}^{\mathrm{n}}\right)$ and $\mathrm{u}_{1} \in \mathrm{H}^{\mathrm{s}-1}\left(\mathbb{R}^{\mathrm{n}}\right)$.

Theorem 4.2. Under the same assumption as in Theorem 4.1, then $u$ given by (2.4) satisfies the decay estimate:

$$
\left\|\partial_{x}^{k} u(t)\right\|_{H^{s+2-2 k}} \leq C I_{0}(1+k)^{-\frac{k-1}{2}}, 1 \leq k \leq\left[\frac{s+2}{2}\right] .
$$

Proof. Let $k \geq 1, m \geq 0$ be integers, In view of (2.4), by using 1) and 3) of Proposition 3.1 with $p=2$, we have that

$$
\left\|\partial_{x}^{k+m} u(t)\right\| \leq\left\|\partial_{x}^{k+m} G(t) * u_{0}\right\|_{L^{2}}+\left\|\partial_{x}^{k+m} H(t) * u_{1}\right\|_{L^{2}}
$$




$$
\begin{aligned}
& \leq C(1+t)^{-\frac{k+m}{2}-\frac{n}{2}\left(\frac{1}{p}-\frac{1}{2}\right)}\left\|u_{0}\right\|_{L^{2}} \\
& +C(1+t)^{-\frac{l_{1}}{2}}\left\|\partial_{x}^{k+m+l_{1}} u_{0}\right\|_{L^{2}} \\
& +C(1+t)^{-\frac{k-1+m}{2}-\frac{n}{2}\left(\frac{1}{p}-\frac{1}{2}\right)}\left\|u_{1}\right\|_{L^{2}}
\end{aligned}
$$

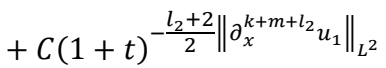

$$
\begin{aligned}
& \leq C(1+t)^{-\frac{k}{2}}\left\|u_{0}\right\|_{L^{2}} \\
& +C(1+t)^{-\frac{k-1}{2}}\left\|u_{1}\right\|_{L^{2}} \\
& +C(1+t)^{-\frac{l_{1}}{2} \| \partial_{x}^{k+m+l_{1}}} u_{0} \|_{L^{2}} \\
& +C(1+t)^{-\frac{l_{2}+2}{2}}\left\|\partial_{x}^{k+m+l_{2}} u_{1}\right\|_{L^{2}} \\
& \leq C(1+t)^{-\frac{l_{1}}{2}}\left\|\partial_{x}^{k+m+l_{1}} u_{0}\right\|_{L^{2}} \\
& +C(1+t)^{-\frac{l_{2}+2}{2}}\left\|\partial_{x}^{k+m+l_{2}} u_{1}\right\|_{L^{2}} \\
& +C(1+t)^{-\frac{k-1}{2}}\left\|\left(u_{0}, u_{1}\right)\right\|_{L^{2}}
\end{aligned}
$$

here $k \geq 1, k+m+l_{1} \leq s+1, k+m+l_{2} \leq s-1$. Choose the minimal integers $l_{1}$ and $l_{2}$ satisfying

$$
\frac{l_{1}}{2} \geq \frac{k-1}{2}, \frac{l_{2}+2}{2} \geq \frac{k-1}{2}
$$

i.e. $l_{1}=k+1, l_{2}=l_{1}-2$, Then we obtain that

$$
\left\|\partial_{x}^{k+m} u(t)\right\|_{L^{2}} \leq C I_{0}(1+t)^{-\frac{k-1}{2}},
$$

with $0 \leq m \leq s+2-2 k$. Take sum with $0 \leq m \leq s+2-$ $2 k$, we obtain (4.3). Thus Theorem (4.2) is proved.

Remark 4.1. Under the same assumption as in Theorem 4.1, $u$ given by (2.4) also satisfies the following decay estimate:

$$
\left\|\partial_{x}^{k} u_{t}(t)\right\|_{H^{s-1-2 k}} \leq C I_{0}(1+k)^{-\frac{k}{2}}, 0 \leq k \leq\left[\frac{s-1}{2}\right] .
$$

If we assume the initial data belong to $L^{1}(\mathbb{R})$, then by using Proposition (3.1) with $p=1$, we have the following sharp decay estimates.

Theorem 4.3. Let $s \geq 1$ be an integer. Assume that $u_{0} \in$ $H^{s+1}\left(\mathbb{R}^{n}\right) \cap L^{1}\left(\mathbb{R}^{n}\right)$ and $u_{1} \in H^{s-1}\left(\mathbb{R}^{n}\right) \cap L^{1}\left(\mathbb{R}^{n}\right)$, and put

$$
I_{1}=\left\|u_{0}\right\|_{H^{s+1}}+\left\|u_{1}\right\|_{H^{s-1}}+\left\|\left(u_{0}, u_{1}\right)\right\|_{L^{1}} .
$$

Then the solution $u$ to (1.1) and (1.2) given by (2.4) satisfies the following decay estimates:

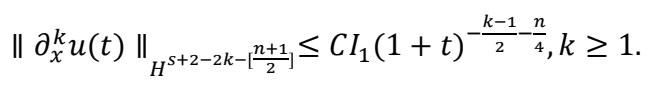

Proof. Let $k \geq 1, m \geq 0$ be integers, In view of (2.4), by using 1) and 3) of Proposition 3.1 with $p=1$, we have that

$$
\begin{aligned}
& \left\|\partial_{x}^{k+m} u(t)\right\| \leq\left\|\partial_{x}^{k+m} G(t) * u_{0}\right\|_{L^{2}}+\left\|\partial_{x}^{k+m} H(t) * u_{1}\right\|_{L^{2}} \\
& \leq C(1+t)^{-\frac{k+m}{2}-\frac{n}{2}\left(\frac{1}{p}-\frac{1}{2}\right)}\left\|u_{0}\right\|_{L^{2}} \\
& +C(1+t)^{-\frac{l_{1}}{2} \| \partial_{x}^{k+m+l_{1}}} u_{0} \|_{L^{2}} \\
& +C(1+t)^{-\frac{k-1+m}{2}-\frac{n}{2}\left(\frac{1}{p}-\frac{1}{2}\right)}\left\|u_{1}\right\|_{L^{2}} \\
& +C(1+t)^{-\frac{l_{2}+2}{2}}\left\|\partial_{x}^{k+m+l_{2}} u_{1}\right\|_{L^{2}} \\
& \leq C(1+t)^{-\frac{k}{2}-\frac{n}{4}}\left\|u_{0}\right\|_{L^{2}} \\
& +C(1+t)^{-\frac{k-1}{2}-\frac{n}{4}}\left\|u_{1}\right\|_{L^{2}}
\end{aligned}
$$

$$
\begin{aligned}
& +C(1+t)^{-\frac{l_{1}}{2}}\left\|\partial_{x}^{k+m+l_{1}} u_{0}\right\|_{L^{2}} \\
& +C(1+t)^{-\frac{l_{2}+2}{2}}\left\|\partial_{x}^{k+m+l_{2}} u_{1}\right\|_{L^{2}} \\
& \leq C(1+t)^{-\frac{l_{1}}{2}}\left\|\partial_{x}^{k+m+l_{1}} u_{0}\right\|_{L^{2}} \\
& +C(1+t)^{-\frac{l_{2}+2}{2}}\left\|\partial_{x}^{k+m+l_{2}} u_{1}\right\|_{L^{2}} \\
& +C(1+t)^{-\frac{k-1}{2}-\frac{n}{4}}\left\|\left(u_{0}, u_{1}\right)\right\|_{L^{2}}
\end{aligned}
$$

here $l_{1} \geq 0, l_{2} \geq-2, k+m+l_{1} \leq s+1,0 \leq k+m+l_{2} \leq$ $s-1$

Choose the smallest integers $l_{1}$ and $l_{2}$ satisfying

$$
\frac{l_{1}}{2} \geq \frac{k-1}{2}+\frac{n}{4}, \frac{l_{2}+2}{2} \geq \frac{k-1}{2}+\frac{n}{4}
$$

It yield that $l_{1}=k-1+\left[\frac{n+1}{2}\right] \quad, \quad l_{2}=l_{1}-2$. Thus $m$ satisfies $0 \leq m \leq s+2-2 k-\left[\frac{n+1}{2}\right]$. Take sum with $0 \leq m \leq s+2-2 k-\left[\frac{n+1}{2}\right]$, we obtain that

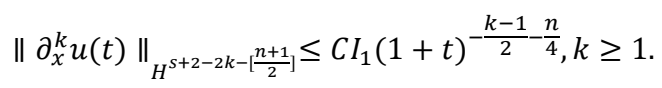

Thus Theorem 4.3 is proved.

Remark 4.2. Apart from the above decay estimates, by similar computation we also have the following estimate :

$$
\left\|\partial_{x}^{k} u_{t}(t)\right\|_{H^{s-1-2 k-\left[\frac{n+1}{2}\right]}} \leq C I_{1}(1+t)^{-\frac{k}{2}-\frac{n}{4}}, k \geq 0 .
$$

Remark 4.3. The estimates in Theorem 4.2 and Theorem 4.3 indicate that the decay structure of solutions to the linear problem (1.1), (1.2) is of regularityloss type.

\section{Acknowledgments}

This work is partially supported by the Fundamental Research Funds for the Central Universities (Grant No. 2018MS052 ).

\section{REFERENCES}

[1] C. R. da Luz and R. C. Charão, Asymptotic properties for a semi-linear plate equation in unbounded domains, J. Hyperbolic Differential Equations, 6 (2009), 269-294.

[2] Y. Sugitani and S. Kawashima, Decay estimates of solutions to a semilinear dissipative plate equation, J. Hyperbol. Di. Eq., 7(2010), 471-501.

[3] Y. Liu and S. Kawashima, Global existence and asymptotic behavior of solutions for quasi-linear dissipative plate equation, Discrete Contin. Dyn. Syst., 29 (2011), 1113-1139.

[4] Y. Liu and S. Kawashima, Decay property for a plate equation with memory-type dissipation, Kinet. Relat. Mod., 4 (2011), 531-547.

[5] Y. Liu and S. Kawashima, Global existence and decay of solutions for a quasi-linear dissipative plate equation, $\mathrm{J}$. Hyperbol. Differ. Eq., 8 (2011), 591-614.

[6] T. Hosono and S. Kawashima, Decay property of regularity-loss type and application to some nonlinear hyperbolicCelliptic system, Math. Models Meth. Appl. Sci.,16 (2006), 1839-1859.

[7] K. Ide and S. Kawashima. Decay property of regularityloss type and nonlinear effects for dissipative timoshenko system, Math. Models Meth. Appl. Sci., 18 (2008),10011025 . 
[8] Y. Liu, The pointwise estimates of solutions for semilinear dissipative wave equation, Commun. Pur. Appl. Anal., 12 (2013), 237-252.

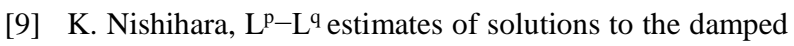
wave equation in 3-dimensional space and their application, Math. Z., 244 (2003), 631649.

[10] K. Nishihara and H.-J. Zhao, Decay properties of solutions to the Cauchy problem for the damped wave equation with absorption, J. Math. Anal. Appl., 313 (2006), 598-610.

[11] W. Wang and T. Yang, The pointwise estimates of solutions for Euler equations with damping in multidimensions, J. Differential Equations, 173 (2001), 410450.

[12] P. M. N. Dharmawardane, J. E. Muñoz Rivera and S. Kawashima, Decay property for second order hyperbolic systems of viscoelastic materials, J. Math. Anal. Appl., 366 (2010), 621-635.

[13] M. Fabrizio and B. Lazzari, On the existence and the asymptotic stability of solutions for linear viscoelastic solids, Arch. Rational Mech. Anal., 116 (1991), 139-152.

[14] Y. Liu and S. Kawashima, Decay property for the Timoshenko system with memory-type dissipation, Math. Models Meth. Appl. Sci., 22 (2012), 1-19.

[15] Y. Liu and S. Kawashima, Global existence and asymptotic decay of solutions to the nonlinear Timoshenko system with memory, Nonlinear AnalysisTMA, 84 (2013), 1-17.

[16] J. E. Muñoz Rivera, M.G. Naso and F.M. Vegni, Asymptotic behavior of the energy for a class of weakly dissipative second-order systems with memory, J. Math. Anal. Appl.,286 (2003), 692-704. 